- European Mobility Scheme for Physics Students - That the Executive Committee consider a Polish Physical Society proposal that it take over the mobility scheme secretariat. - That students participating in the scheme be offered one-year's membership free-of-charge.

- Professional Qualifications - To approve the Register Commission's proposal that the Executive Committee considers providing financial support from EPS to cover the fees of applicants from less-favoured regions.

- That the Commission considers changing the currency of the registration fee to the ECU.

- Internet Coordination Group - That the ICG makes proposals to the Executive Committee on the possibility of having Division and Group membership lists available on Internet.
- Divisions - To have a final agreement for the an EPS-European Astronomy Society Joint Division of Physics and Astronomy ready for approval by the Societies' Councils in 1997.

- Next Meeting - To hold the next Council meeting in Mulhouse on the announced dates (21-22 March 1997). The 1998 meeting would then be in Holland (on 27-28 March).

- Executive Committee - To elect the following: President: H. Schopper, Geneva; PresidentElect: D.L. Weaire, Dublin; Secretary: J.-P. Ansermet, Lausanne; Vice-Secretary: C.

Sébenne, Paris; Treasurer: J.L. Lewis, Malvern; Vice-Secretary: E. Osnes, Oslo; Members: S.

Bagayev, Novosibirsk; G. Benedek, Milan; C.M. Ferreira, Lisbon; G.C. Morrison, Birmingham; M.J. van der Wiel, Nieuwegian.

\title{
Many Opportunities for Collaboration
}

The EPS Internet Coordination Group (ICG) and the EPS Publications Committee ad hoc Discussion Group for Online Publications organized a discussion meeting in Paris on 26 February to review developments in electronic communication in science, current activities carried out under the EPS EurophysNet umbrella, and plans for future action. The meeting was divided into sessions on indexing, electronic publications and information services to reflect EPS initiatives involving Oldenburg University's PhysNet index services, the EurophysNet information service supervised by the ICG and the deliberations of the Publications Committee, part of which involve a Task Force on Electronic Publishing. In a short introduction, Franck Laloë (ENS Paris) who chairs the Publications Committee, spoke of the pressure from colleagues to exploit electronic means of communication and to consolidate the proliferating sources of information. He felt that the EPS was in a good position to act, at least as a coordinator. WWW URLs of the services described below are available in europhysicsnews extra (March-April 1996) on EurophysNet at http://epswww.epfl.ch.

\section{PhysDep Expansion}

The first pilot project to be operated under the EurophysNet umbrella, namely the PhysDep indexing service, was reviewed by Eb Hilf (Oldenburg University). PhysDep allows one to search for information (research topics, fax numbers, teaching materials, etc.) found on the WWW pages of European Physics Institution Servers. The service is in the process of being upgraded by ensuring that the indexed pages are authorised by institutions and by encouraging the use of tags and keywords to improve the indexing. It was felt at the meeting that the authorization procedure needed top-down action as well as the bottom-up identification of sources using search engines. The ICG has therefore recently asked national physical societies to nominate National WWW Coordinators who would help ensure that physics institutions name authorised WWW server sites which they then make known to PhysDep.

Eb Hilf envisaged a series of index services for various topics, each operating under the PhysNet banner and collaborating with groups specialising in particular topics. Like PhysDep, they could be super-

Oldenburg's PhysNet team in action. From the left, Heinrich Stamerjohanns, Eb Hilf and Thomas Severiens. PhysNet operates, under the EPS World-Wide Web EurophysNet umbrella, a series of indexes (PhysDep, PhysDoc, PhysDis) based on the Harvest search engine. vised by EPS under the EurophysNet umbrella, and perhaps carry the EPS logo as long as they fulfil set standards.

\section{TIPTOP Collaboration}

A group based at the Technical University of Vienna maintains a list of physics servers as part of a well-known collection of useful physics links called Physics Around the World (PAW). The site's developers (Mikko Karttunen, a student at McGill University in Canada, and Günther Nowotny from the TU Vienna) agreed at the meeting that PAW would collaborate with PhysDep to create a single list PhysNet of physics institution servers. It will be mirrored at The Internet Pilot TO Physics (TIPTOP) to which $P A W$ now belongs.

TIPTOP is a new "world-wide, onestop, knowledge-server", and Mikko Karttunen described how it took shape in the early 1990 s when he and Nowotny created PAW. The TIPTOP team has automated the laborious work of running a state-of-theart global information service over WWW

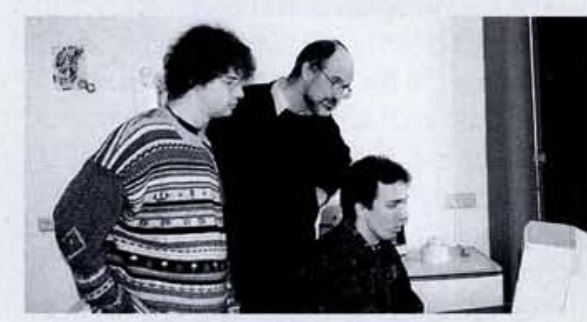

using a dedicated server in Sweden. The MIT-based Net Advance of Physics has joined the project, bringing a subject index to archival literature as well as a US site.

TIPTOP operates sophisticated upload facilities that allow users to input information that can then be made available in a systematic fashion over the WWW. It was agreed at the meeting that the complementary PhysDep and TIPTOP upload facilities will be coordinated to ensure that physics institutions have a highly visible entry point for updating the institution list.

\section{PhysDoc and PhysDis}

PhysNet launched late last year a second service, called PhysDoc, which indexes documents stored on PhysDep servers. Thomas Severiens, a student from Oldenburg University who helped establish PhysDep, outlined the reasons for selecting the Harvest search machine (it is one of the best available and lies in the public domain). In the case of PhysDoc, indexed information may be either full texts or bibliographic information, depending on how an author stores a document locally. PhysDoc complements the centralized e-print bulletin board system based at the Los Alamos National Laboratory (LANL), especially for fields where no e-print slots exist and for non-permanent documents, large graphic-oriented papers, annual reports, and scanned-in texts.

In order to enlarge PhysDoc's coverage beyond the present 3000 documents stored in servers based at 70 European institutions, the ICG is in the process of contacting physics institutions through the national coordinators to ask them to make sure that the document archives are known to PhysDoc. PhysNet is also planning to enlarge the scope by establishing an index (PhysDis) for $\mathrm{PhD}$ theses. In this case, authenticity is extremely important and has to be guaranteed by institutions. It is envisaged that the national coordinator scheme will lead to the necessary authenticity, and that PhysDis will also operate under the PhysNet banner as a EurophysNet service supervised by the ICG.

It is also necessary to improve PhysDoc and PhysDis indexing by ensuring that the search engines identify relevant documents. Information providers can help by using a short list of metatags or keywords. Paul Ginsparg (LANL) suggested that indexing information contained in the headers of PostScript files can also be accessed. It was therefore agreed that physics institutions would be asked by the ICG through national coordinators to incorpo- 
rate metatags, keywords or PostScript header information to denote basic indexing items such as authors, titles, etc.

\section{Links to Indexes}

Several major institutions and commercial databanks and publishers maintain indexes of specialised literature and/or substantial collections of electronic documents stored on-line. It is important to see if these sources can be integrated into the PhysDoc index without excessive effort. In the case of institutions, this should be reasonably straightforward if resources can be found to set up scripts that pass queries from the PhysDoc query interface to the institution index. It was therefore interesting to review institutional indexes.

Hartmut Preissner from DESY, Hamburg, described the Stanford Linear Accelerator Center's SPIRES high-energy physics index that has been mirrored at DESY since 1973. The index is extremely comprehensive, but labour intensive and further effort is needed to convert it into a modern, automatically loaded, WWW-compatible database system. However, while extension of the SPIRES system into other fields seemed unlikely, it appeared that a query interface to PhysDoc could be implemented.

Michael Draper described how CERN is extending its WWW preprint server by interfacing it to the organization's library database system ALEPH. After this is established it should be possible to see how the query interface could be linked to PhysDoc. Once again, the CERN approach is unique and poorly adapted for more general use.

Only a few projects have developed ways to pass WWW search queries between different systems. However, the TH Darmstadt has pioneered techniques to search for documents stored in the servers of several German libraries. This experience suggests that PhysDoc could be interfaced to other indexes without too much effort.

\section{General Information}

Kenneth Holmlund described how TIPTOP has introduced new features which will doubtless soon become as popular as $P A W$. The most important are a "living encyclopedia" of physics (an on-line encyclopedia maintained by authors and editors), a "virtual laboratory" with interactive online experiments, a global bulletin board for announcements, and an online archive of $\mathrm{PhD}$ theses. Discussion focussed on how TIPTOP could be collaborate with physical societies and with EurophysNet in these areas. In addition to PhysDis, Oldenburg's PhysNet team, in collaboration with
From the left, Günther Nowotny, Mikko Kartunen and Kenneth Holmlund, founder members of TIPTOP who partictpated at the EPS discussion meeting. Kenneth and Madelen Holmlund from the University of Umeå, Peter Berglund from the Helsinki University of Technology, and Risto Nieminen from the Centre for Scientific Computing in Finland recently joined TIPTOP, along with Karen Keck and Norman Redington from the MIT-based Net Advance of Physics.

a group from Kiel University, has in mind a PhysEd indexing service for educational materials. So interaction with TIPTOP in the education area seemed possible.

TIPTOP's global bulletin board also offered interesting possibilities for collaboration, in addition to maintaining the institution list. It was agreed that TIPTOP would provide a EurophysNet upload facility for conference organizers wishing to place announcements on WWW. It was also envisaged that the EurophysNet Europhysics conference list would be merged into the bulletin board to give PhysCal, a single physics calender. Eventually, one could envisage doing the same for job openings (EurophysNet maintains a list of pointers to lists of job openings and jobs announcements can be uploaded into TIPTOP ).

\section{Electronic Publications}

Electronic preprints and prepublications obviously represent a key aspect of communication in science so it was very interesting to hear Paul Ginsparg's opinions during his review of the status of the renowned Los Alamos e-print server with its 36000 accesses each day. The server has been mirrored at SISSA in Trieste since 1992 and at Augsburg University starting in 1993; an Australian mirror takes to the air shortly and discussions are underway to establish mirrors in Brazil, France, Russia, and Sweden. An important recent innovation is an upload filter to ensure that stored $\mathrm{T}_{\mathrm{E}} \mathrm{X}$ documents are reasonably homogeneous. Ginsparg agreed at the meeting that one could envisage a consortium of European preprints servers based on the Trieste and Augsburg sites, and on others which could be rapidly put into operation in Paris and Umeå. One possibility is to coordinate activities under an EPS/EurophysNet umbrella and logo - an approach that would guarantee long-term stability, security and accessibility. Other countries could be included in the future, and the framework could provide a base for expansion into fields not covered so far. One could also organize experiments involving such things as indexing and storage that would eventually provide add-on services offering secure archival, links to publishers, the-

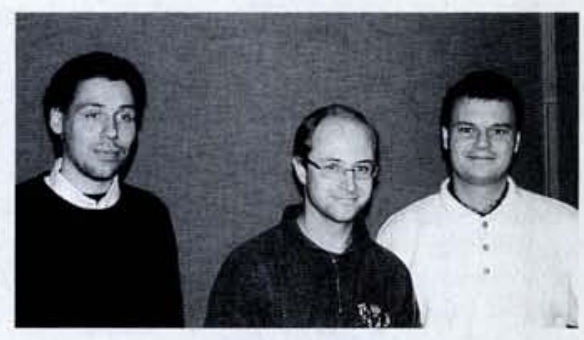

sauri, sophisticated search engines, systems for annotating texts, etc. One such service could be a clearing house to mark up texts prior to transmittal to publishers. Hans Sens, who has been much involved in promoting the use of standard mark-up to ensure flexible, robust and durable archiving, presented a proposal along these lines.

The framework may also be appropriate for evaluating on-line peer review - a subject which was not directly addressed at the meeting. However, it emerged that an interesting experiment is planned in highenergy physics involving a fully electronic journal, including refereeing by the usual techniques. The American Physical Society has apparently expressed interest and offered encouragement. The EPS could perhaps be involved as well by, for instance, asking its High Energy and Particle Physics Division to propose coeditors, as is already the case for Europhysics Letters.

Publishers clearly have an interest in such developments so it was useful to learn about the status of electronic publishing as seen from their standpoint. Many larger publishers are producing, or will soon produce, electronic versions of journals in parallel with printed versions. Smaller publishers, including those in eastern Europe, are considering this possibility.

One interesting aspect of electronic publishing that is often overlooked is historical archiving. Walther Bollman from Jena University described a project that is archiving, on CD ROMs, Annalen der Physik 1-20 (1999-1805) as well as papers published in Annalen der Physik and Z. Physik in 1920-24. It follows on from a project that archived in the FIZ Karlsruhe PHYS database some 2000 papers published in $1900-$ 78. Discussion naturally centred on the possibility of having such archives available over WWW and integrated into a larger information system.

The future in a time of rapid change may not be foreseeable. The discussion meeting demonstrated that one nevertheless has not only the duty but also the opportunity to cooperate and collaborate coherently as a single distributed group in establishing a web of integrated services.

E.R. Hilf, P.G. Boswell, F. Laloë 